\title{
Genomic Sequencing and the Impact of Molecular Diagnosis on Patient Care
}

\author{
Benjamin D. Solomon ${ }^{\mathrm{a}-\mathrm{c}}$ \\ ${ }^{a}$ Division of Medical Genomics, Inova Translational Medicine Institute, ${ }^{b}$ Department of Pediatrics, Inova Health \\ System, Virginia Commonwealth University (VCU) School of Medicine, Falls Church, Va., and ${ }^{\mathrm{C}}$ Medical Genetics \\ Branch, National Human Genome Research Institute, Bethesda, Md., USA
}

\begin{abstract}
Evolving sequencing technologies allow more accurate, efficient and affordable genomic analysis. As a result, these technologies are increasingly available, especially to provide molecular diagnoses for patients with suspected genetic disorders. However, there are many challenges to using genomic sequencing to benefit patients, including concerns that there is insufficient evidence that identifying an underlying molecular explanation may positively impact a patient's healthcare. This concern has many repercussions, including funding and/or (in some countries and healthcare systems) insurance reimbursement for genomic sequencing. To investigate this concern, all monogenic disorders were analyzed based on the impact of achieving molecular diagnosis. Of the 2,849 individual genes in which germline mutations cause disorders (not including contiguous gene syndromes or what may be categorized as susceptibility alleles), our analyses showed a specific, available intervention related to at least one affected organ system for 1,419 (49.8\%) genes. In $95.6 \%$ of these genes, the intervention(s) would be recommended during the pediatric time frame.
\end{abstract}

(c) 2015 S. Karger AG, Basel

Evolving technologies allow genomic sequencing to be performed more accurately, efficiently and affordably [Biesecker and Green, 2014; Hayden, 2014]. Due to these advances, genomic sequencing is becoming more widespread. One major use of genomic sequencing is as a tool to provide a molecular diagnosis for conditions that may (c) 2015 S. Karger AG, Base

$1661-8769 / 15 / 0061-0004 \$ 39.50 / 0$ be due to genetic causes. However, there are many challenges to using genomic sequencing to benefit patients. [Dewey et al., 2014] One concern is that there is insufficient evidence that identifying a molecular explanation for a disease will positively impact health. This has many repercussions, including funding and/or (in some countries and healthcare systems) insurance reimbursement for genomic sequencing [Steenhuysen, 2014].

Before addressing the above point about health impacts of providing a molecular diagnosis, some background information may be helpful. 'Genomic sequencing' in this context refers both to exome sequencing, which focuses on the protein-coding regions of the genome (about $1 \%$ of the genome, but which appears to contain the majority of disease-causing mutations), and whole-genome sequencing. Genomic sequencing can provide explanations for patient presentations by interrogating many genes and other loci simultaneously [Biesecker and Green, 2014]. In the research realm, in the last year, mutations in over 150 individual genes have been newly found to cause genetic disorders [Solomon et al., 2013]. Almost three-quarters of these were identified through genomic sequencing. In approximately half, genomic sequencing was used alone without separate, preliminary steps to help hone in on the genomic region harboring the causative mutations [Solomon, 2014].

Beyond the excitement of providing novel explanations for medical conditions, genomic sequencing can help diagnose conditions that are not clinically obvious even to an experienced geneticist or other clinician. Along these lines,

\section{KARGER 125}

E-Mail karger@karger.com www.karger.com/msy
Benjamin D. Solomon, MD

Department of Pediatrics, VCU School of Medicine Inova Campus 3300 Gallows Road, 2nd Floor, Claude Moore Building Falls Church, VA 22042 (USA)

E-Mail benjamin.solomon@inova.org 


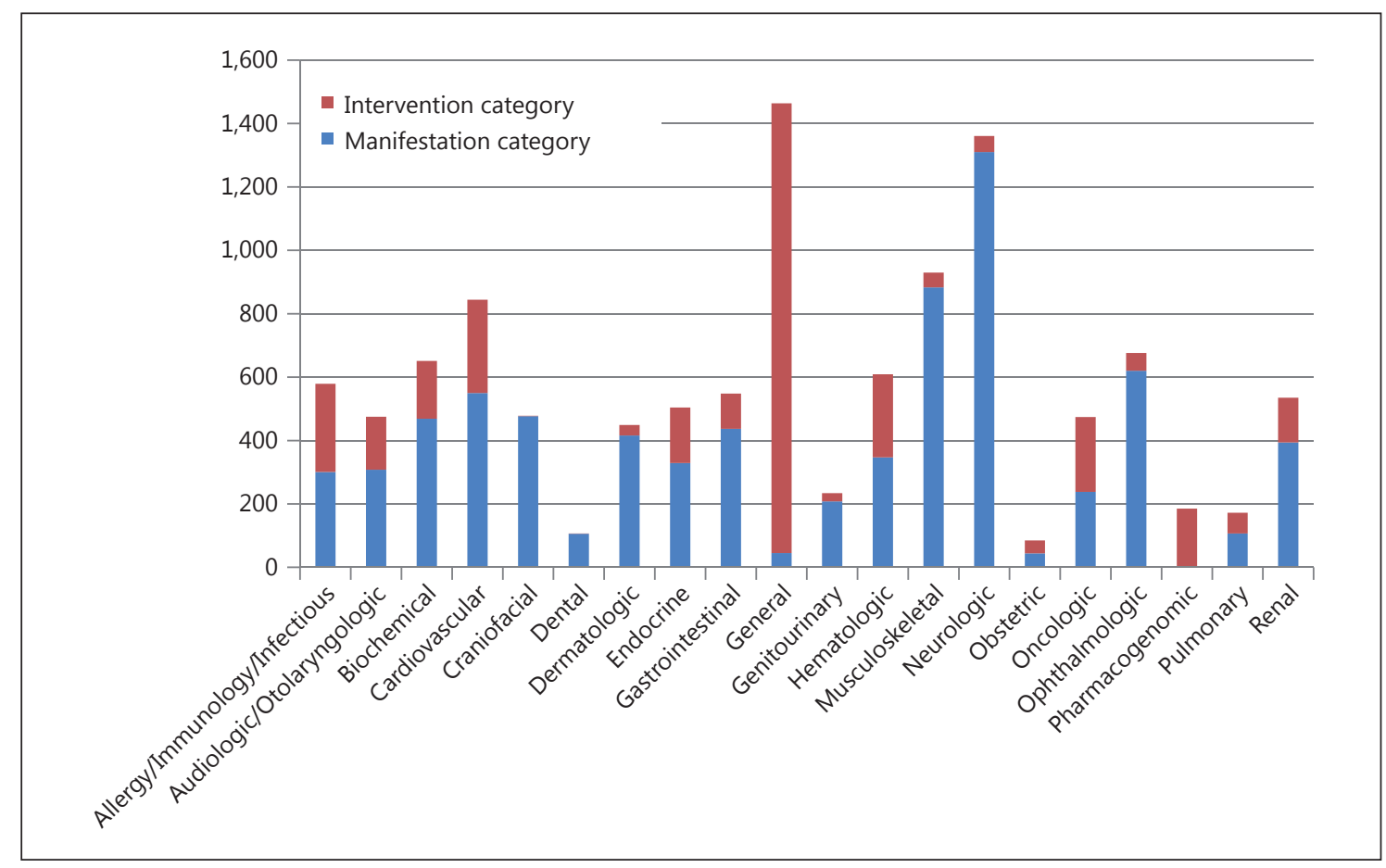

Fig. 1. Clinical management categorization of all known germline single-gene disorders. Each of the 2,849 single-gene disorders due to germline mutations were individually, manually classified by organ systems. Organ systems affected (manifestation category) by mutations in that gene are shown in blue. Organ systems/ categories with available, effective interventions such that early/ molecular diagnosis would be judged to be beneficial are shown in red (intervention category). Genes for which there was not judged to be specific benefit from early/molecular diagnosis were binned into the 'general' intervention category and are also shown in red. Categories are listed on the $\mathrm{x}$-axis; the $\mathrm{y}$-axis represents the number of genes per category. As many genetic disorders affect multiple organ systems, a single gene may involve multiple categories. genomic sequencing increasingly identifies non-classically affected patients (i.e. with atypical or subtle disease presentations). Recent publications have described an overall $\sim 25 \%$ success rate using exome sequencing to provide a molecular diagnosis for a patient presentation; higher rates have been described with certain subcategories of disease [Yang et al., 2013; Biesecker and Green, 2014]. Though direct comparisons are difficult, these rates appear superior to traditional genetic diagnostic approaches; further studies are required for comparison.

Related to high-profile research successes, commercial genomic sequencing is growing considerably. Clinical exome sequencing was first offered in the fall of 2011. Within the next $\sim 3$ years, over 30 laboratories, representing 12 separate countries, offered commercial exome sequencing; 10 laboratories now offer commercial wholegenome sequencing. New genomic sequencing products are being launched at the rate of one new offering per month, and there are almost 40 commercially available genomic sequencing products available in the US alone (http://www.ncbi.nlm.nih.gov/gtr/labs; nxt.gd/1nPuv4u). The direct cost of genomic sequencing is a small fraction of the price even a few years ago. However, the larger cost involves analysis of the vast amounts of genomic data [Biesecker and Green, 2014]. The clinical charge for exome and genome sequencing ranges from approximately USD $4,000-15,000$. Cost differences involve factors such as whether sequencing is done only on the affected patient or also includes family studies, as latter approach is often more informative. Advertised turn-around times vary from several weeks to 5 months (nxt.gd/ $1 \mathrm{nPuv} 4 \mathrm{u}$ ).

In addition to considering price and success rate (both of which are anticipated to continue to improve), there is the question of whether and how using genomic sequencing to find genetic explanations impacts clinical care. We believe there are fundamental misperceptions about the effects of molecular diagnosis, largely due to lack of overall analyses.

In general, and regardless of the disorder, knowledge of the genetic cause of a medical condition may be beneficial 
related to issues such as informed medical decision making, selection of optimal supportive care, prognostic considerations, and avoidance of unnecessary testing. A counter-argument is that, while there are some highly publicized cases in which establishing a molecular diagnosis did indeed provide a life-saving or other dramatic change in patient management, in most situations, there is not enough impact on medical care to justify the test. This belief may be because the rarity and medical impact of many genetic disorders makes it very difficult to implement traditional double-blind placebo-based cohort studies in the vast majority of conditions. However, systematic inquiries based on the literature are also largely lacking.

Our analyses question the argument that molecular diagnosis infrequently impacts patient care in ways other than the general benefits mentioned above.

By June, 2014, 2,849 individual genes had been identified and included in databases of known causes of germline genetic disorders: Online Mendelian Inheritance in Man (OMIM, http://www.ncbi.nlm.nih.gov/omim) and the Clinical Genomic Database (CGD, http://research.nhgri. nih.gov/CGD/). These databases typically lag 1-2 months behind newly published genetic findings. The CGD was specifically designed to examine, for each disorder, the clinical utility of molecular diagnosis and the availability of specific medical interventions. To accomplish this, all conditions were individually/manually reviewed by a single clinical geneticist. Consideration for there being a specific advantage to molecular diagnosis was based on the following requirements: (1) the condition must be clinically significant (involve some degree of morbidity or mortality); (2) there must be available, potentially beneficial intervention(s) (these could include preventive measures, surveillance, or medi$\mathrm{cal} /$ surgical treatments), and (3) there must be a benefit to early molecular diagnosis versus diagnosis on clinical grounds, such that the efficacy of these interventions would be diminished with later diagnosis. Details about the interventions for each gene/condition are available in the CGD website, which is updated regularly to keep pace with scientific and medical progress [Solomon et al., 2013].

Aggregate results are shown in figure 1. Of the 2,849 genes in which germline mutations cause disorders (this does not include contiguous gene syndromes or what are considered susceptibility alleles), there was noted to be a specific, available intervention related to at least one affected organ system for $1,419(49.8 \%)$ genes. In creating the CGD, the general age group (pediatric vs. adult, with 18 years of age considered as the cut-off between the 2 groups) at which interventions would be warranted was analyzed based on the literature reports regarding the age of onset and recommended interventions (including surveillance) for manifestations of these conditions. For $1,357(95.6 \%)$ of the genes in which interventions were judged to be warranted if mutations were found in the corresponding genes, the interventions would be recommended during the pediatric time frame.

These judgments are admittedly highly subjective determinations that require more investigation, despite challenges involved in producing sufficient evidence related to many of the conditions. Additionally, some conditions are relatively clinically obvious and would not require genomic sequencing for diagnosis. However, the overall conclusion is that determination of the molecular cause of a large proportion of genetic conditions can alter patient care and may have immediate clinical benefit. We hope that considerations such as funding and insurance reimbursement policies will take this conclusion into account.

\section{Acknowledgements}

This work was supported by the Inova Translational Medicine Institute, Inova Health System in relation to salary support for the author. Data on commercial offerings for genomic sequencing were supplied by Jud Schneider, $\mathrm{PhD}(\mathrm{NextGxDx})$, who has given written permission for this inclusion and did not receive any compensation.
References
-Biesecker LG, Green RC: Diagnostic clinical genome and exome sequencing. $\mathrm{N}$ Engl $\mathrm{J}$ Med 370:2418-2425 (2014).

-Dewey FE, Grove ME, Pan C, Goldstein BA, Bernstein JA, et al: Clinical interpretation and implications of whole-genome sequencing. JAMA 311:1035-1045 (2014)

Hayden EC: Technology: The \$1,000 genome. Nature 507:294-295 (2014).

-Solomon BD: Obstacles and opportunities for the future of genomic medicine. Mol Genet Genomic Med 2:205-209 (2014).
- Solomon BD, Nguyen AD, Bear KA, Wolfsberg TG: Clinical genomic database. Proc Natl Acad Sci USA 110:9851-9855 (2013).

Steenhuysen J: As sequencing moves into clinical use, insurers balk. Reuters. June 19, 2014. http://www.reuters.com/article/2014/06/19/ us-health-sequencing-insight-idUSKBNOEU16S20140619.

- Yang Y, Muzny DM, Reid JG, Bainbridge MN, Willis $\mathrm{A}$, et al: Clinical whole-exome sequencing for the diagnosis of mendelian disorders. $\mathrm{N}$ Engl J Med 369:1502-1511 (2013). 